\title{
Penetrating injury to Zone II of the neck causing laryngotracheal injury following a firecracker blast: first reported case
}

\author{
Mohamed Rifzan Jameel, Kuda Banda Galketiya, Thilina M Samarasinghe \\ Teaching Hospital,Peradeniya, Sri Lanka
}

Key words: Transthoracic oesophagotomy; oesophageal foreign body

\section{Introduction}

Penetrating injuries to the neck, are wounds extending deep to the platysma. They comprise $5-10 \%$ of all cases presenting as trauma to the emergency department and is a significant cause of mortality [1]. The incidence of laryngotracheal trauma is 1 in 30,000 admissions to emergency departments [2]. The management of neck injuries needs a quick assessment of the adequacy of airway, breathing and circulation and further management once the patient is stabilized [1].

We discuss a case of an unusual injury to the airway following a firecracker blast.

\section{Case presentation}

A 58-year-old male presented to the Emergency treatment Unit following a penetrating injury to the neck due to the repulsion of a domestic firecracker made using wood and gunpowder. On admission (40 minutes after the accident) he had a wound on the anterior aspect of the neck. He had stridor and laboured breathing. He had a respiratory rate of 20 breaths/minute. His pulse rate was $80 /$ minute and blood pressure $125 / 83 \mathrm{~mm} \mathrm{Hg}$. The patient did not have subcutaneous emphysema or an expanding haematoma in the neck. On examination of the wound, the trachea was severed and the patient was breathing through the defect. Due to the risk of aspiration, urgent endotracheal intubation was done under laryngoscopic guidance by bridging the two ends the trachea and his airway was secured. Emergency exploration was done under general anaesthesia. During exploration, the haematoma was evacuated. There were no major bleeders apart from oozing from the traumatized soft tissue and they were controlled with electro cautery. The thyroid cartilage was shattered into many pieces. The upper part of the trachea was also damaged. Pieces of wood (parts of the domestic firecracker) were found within the wound. There was no major vascular injury. A tracheostomy was made below the original wound and a nasogastric tube was also placed. The

Correspondence: Mohamed Rifzan Jameel

E-mail: rifzan@yahoo.com

Received: 14-11-2018 Accepted: 25-12-2018

(iD) http://orcid.org/0000-0002-6855-7054 DOI: http://doi.org/10.4038/sljs.v36i4.8558 wound was closed with a corrugated rubber tube in situ. The patient was managed in the ICU for three days and was transferred back to the ward. A CT scan done postoperatively showed hyoid bone and thyroid cartilage fractures.

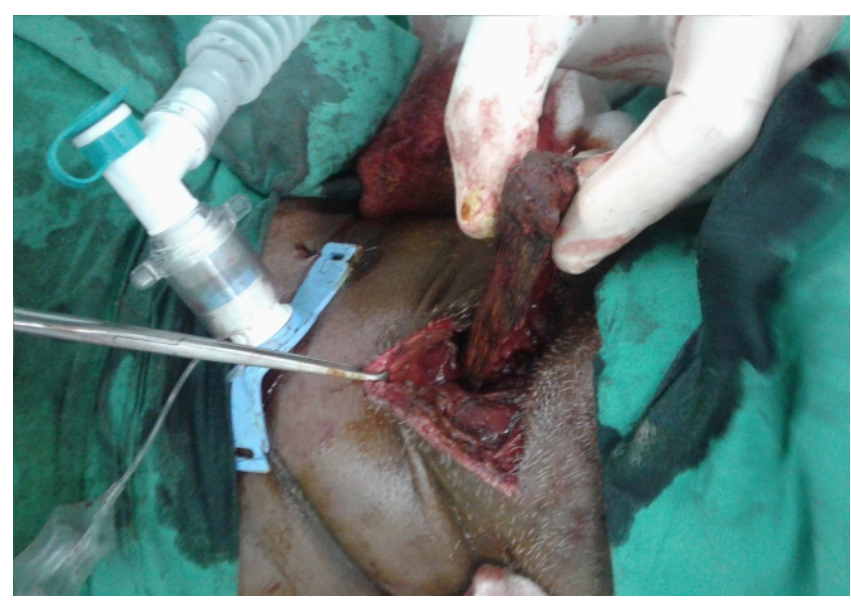

Figure 1. Wooden foriegn body within the wound

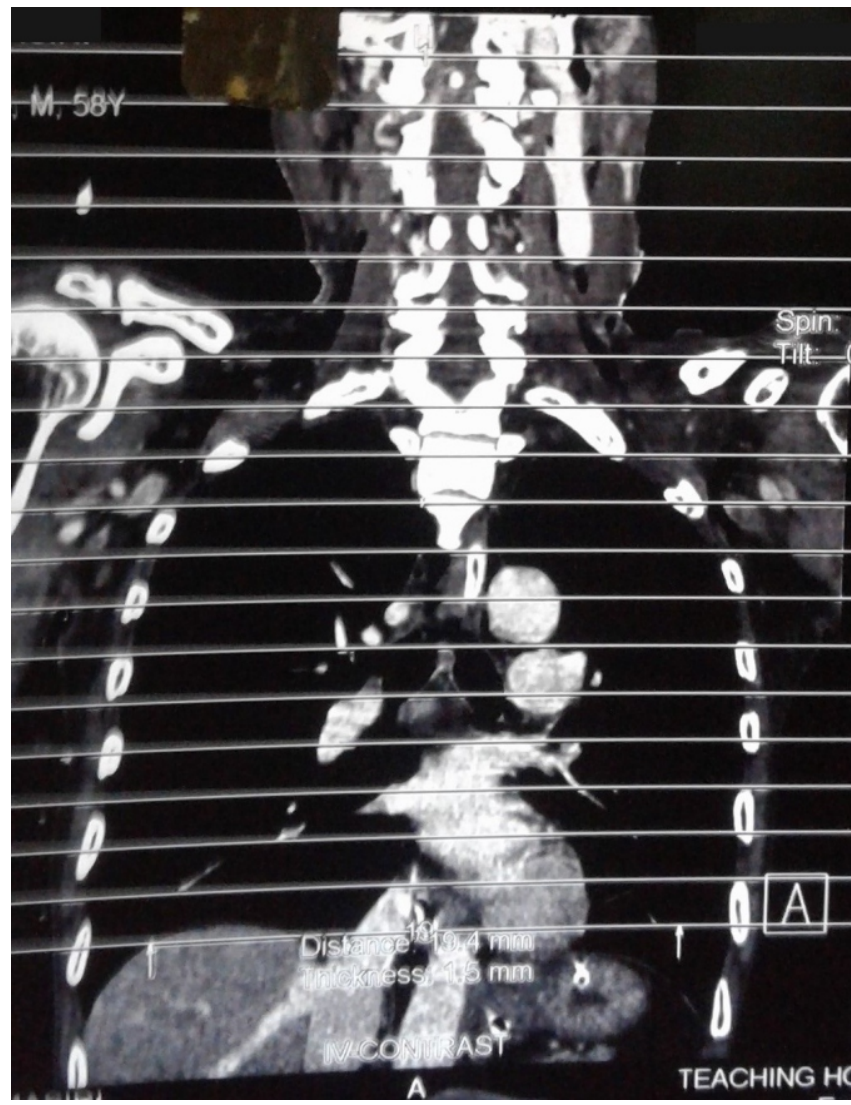

Figure 2. CT chest image showing shattered laryngeal 
The cervical vertebrae were normal. He was then transferred to an ENT unit for further management. The patient has been subsequently discharged with the tracheostomy with a plan for the delayed reconstruction of the laryngeal and tracheal cartilages. Reconstruction of the larynx and trachea was done at the ENT unit, 3 months after the initial injury and followed up monthly.

\section{Discussion}

Firecrackers have been part of civilization for a long time and mainly used in celebration of festivals. Due to its loud noise, it is also used by farmers to scare off animals which are a potential threat to the crops. The explosion has several detrimental effects such as burns, trauma to the eyes, and can cause deafness. The patient discussed had a penetrating injury to the Zone II of the neck causing laryngotracheal damage, with a threat to airway and breathing.

Penetrating neck injuries can present a difficult diagnostic and therapeutic dilemma. Their evaluation and management remain controversial [6]. Broadly the management can be classified into non-operative and operative. Advantages of non-operative management are avoidance of major surgery, but the patient needs to be under constant close monitoring. Whereas operative management deals with life-threatening complications.

Injury to the neck can be defined in three anatomical zones. Zone $\mathrm{I}$ is the area between the clavicle and the cricoid cartilage, Zone II is between the inferior margin of the cricoid cartilage and the angle of the mandible and Zone III is the area between the angles of the mandible to the base of the skull. The commonest are the Zone II injuries (50-80\%) [3].

Prompt examination of patients with suspected laryngeal trauma is essential to identify those who require urgent airway intervention [1]. The goal is always to obtain and maintain a secure airway. Immediate intubation is necessary for patients with respiratory distress or increasing stridor. These patients should have a low threshold to be intubated and the fibre optic bronchoscope can be used in difficult cases. Signs of airway compromise such as minor endolaryngeal lacerations, hematomas and abrasions may be observed in bronchoscopy [6]. our patient had a formal endotracheal tube insertion bridging the two cut ends of the trachea, in the emergency treatment unit.

Airway rupture extending beyond the carina should be managed differently. Single lung ventilation is provided by inserting an endotracheal tube to the main bronchus opposite to the side of injury, under bronchoscopic vision. The injured side should be blocked by the insertion of a bronchial blocker. An intercostal tube is essential, to prevent the development of tension pneumothorax on that side. After the establishment of a secure airway, the patient can undergo further investigations to decide on subsequent management. This will depend upon the mechanism and site of injury, and presence of associated injuries [6].

Symptoms and signs of laryngeal trauma such as hoarseness, stridor, haemoptysis, subcutaneous emphysema, loss of normal thyroid prominence, deviation of the larynx, loss of laryngeal crepitus and external injuries to the neck should be assessed as part of a secondary survey [4]. For stable patients imaging and diagnostics include CT imaging, flexible laryngoscopy, direct laryngoscopy and tracheoscopy [4].

Injury to the great vessels following penetrating injury will be indicated by a haemodynamically unstable patient with either external bleeding from the wound or expanding haematoma of the neck. Distal pulses and presence of bruits should be checked in patients with of neck injuries. Angiography can be done even in stable patients prior to exploration where significant damage to vessels is suspected [4]. Injuries to the upper digestive tract present as bleeding from the mouth, drooling, and subcutaneous emphysema. Contrast-enhanced study of the upper tract will confirm this further. [4]. Our patient did not have any clinical evidence of major vascular or upper digestive injuries.

Indications for mandatory exploration of the neck following penetrating neck trauma are expanding haematoma, active bleeding from the wound, haemodynamic instability and hard aerodigestive signs such as bubbling, hoarseness and salivary fistula [4]. Other patients, following appropriate investigations to exclude injuries, can be safely observed. Our patient had hard signs of tracheal injury and underwent exploration.

The use of firecrackers as a tool to scare off animals has detrimental effects such as near mortality as in this case. Commonly seen injuries are ocular and upper limb injuries. Penetrating injuries to the neck is rather uncommon. Adherence to safety measures when using explosives cannot be overemphasized. It is the duty and responsibility of the health care workers to bring to light such cases as a measure to prevent this happening in the future. It is also the duty of the officials to control these practices by farmers and propose alternative solutions.

\section{Conclusions}

Firecrackers used by farmers to wade off animals can cause near-fatal injuries to the neck and measures should be taken to alleviate this practice and find alternative solutions. However such injuries should be managed promptly and actively to be life-saving.

All authors disclose no conflict of interest. The study was conducted in accordance with the ethical standards of the relevant institutional or national ethics committee and the Helsinki Declaration of 1975, as revised in 2000 . 


\section{References}

1. Pandurangarao SU, Basavaraj P, Yempalle SB, Karnam L. Primary Management and Outcome - Open Laryngotracheal Trauma. Journal of Clinical and Diagnostic Research : JCDR. 2017;11(2):MR01-MR04. https://doi.org/10.7860/JCDR/2017/24155.9321.

2. Schaefer S D. The acute management of external laryngeal trauma. A 27-year experience.Arch Otolaryngol Head Neck Surg. 1992 Jun;118(6):598-604.

3. Varghese A. Penetrating Neck Injury: A Case Report and Review of Management. The Indian Journal of Surgery. 2013;75(1):4346. https://doi.org/10.1007/s12262-012-0531-7.
4. Schaefer, S. D. (2014), Management of acute blunt and penetrating external laryngeal trauma. The Laryngoscope, 124: 233-244. doi:10.1002/lary.24068

5. Gupta B, Gulati A, Gupta D. A Rare Presentation of Pellet Injury in the Neck. ISRN Surgery. 2011;2011:306126. . https://doi.org/10.5402/2011/306126.

6. Philicia Moonsamy, Uma M. Sachdeva, and Christopher R. Morse Management of laryngotracheal trauma Ann Cardiothorac Surg. 2018 Mar; 7(2): 210-216.

\section{Learning Points:}

- Firecracker blast can cause unusual injuries such as laryngotracheal injuries

- Prompt emergency management can save lives

- Society should be educated about the dangers of firecrackers 